\title{
SIERPIŃSKI PEDAL TRIANGLES
}

\author{
JIU DING, L. RICHARD HITT, AND XIN-MIN ZHANG
}

\begin{abstract}
We generalize the construction of the ordinary Sierpiński triangle to obtain a two-parameter family of triangles we call Sierpiński pedal triangles. These triangles are obtained from a given triangle by recursively deleting the associated pedal triangles in a manner analogous to the construction of the ordinary Sierpiński triangle. We study the fractal dimension of these Sierpiński pedal triangles and related area ratios. We also provide some computer generated graphs of the fractals.
\end{abstract}

\section{PRELIMINARIES}

Let $T_{0}$ be a triangle with inner angles $A_{0}, B_{0}$, and $C_{0}$. The pedal triangle of $T_{0}$, denoted by $T_{1}$, is the triangle obtained by joining the feet of the three altitudes of $T_{0}$. Denote the inner angles of $T_{1}$ by $A_{1}, B_{1}$, and $C_{1}$. If $T_{0}$ is an acute triangle, then $T_{1}$ is inscribed inside $T_{0}$. If $T_{0}$ is an obtuse triangle, then two of the vertices of $T_{1}$ will fall on extensions of sides outside of $T_{0}$. If $T_{0}$ is a right triangle, then $T_{1}$ degenerates to a line segment. These cases are illustrated in Figure 1.

It is well known that when $T_{0}$ is not a right triangle, then the original triangle $T_{0}$ is similar to $\triangle A_{0} B_{1} C_{1}, \triangle A_{1} B_{0} C_{1}$, and $\triangle A_{1} B_{1} C_{0}$. We briefly outline the proof here since we are going to use this result to verify some important formulas later. We consider acute triangles only, since the same argument works for the obtuse triangles as well. From Figure 1(a) we see that to show $\triangle A_{0} B_{1} C_{1}$ is similar to $T_{0}$, it suffices to verify that $\angle B_{0}=\angle A_{0} B_{1} C_{1}$. Let $E$ be the point of intersection of the three altitudes of $T_{0}$ (called the orthocenter), then it is easy to see that $\angle B_{0}$ is the same as $\angle A_{0} E C_{1}$, and the latter equals $\angle A_{0} B_{1} C_{1}$ because the quadrilateral $A_{0} C_{1} E B_{1}$ can be inscribed in a circle in which $\angle A_{0} E C_{1}$ and $\angle A_{0} B_{1} C_{1}$ subtend the same arc. A similar argument applies to the similarity between $T_{0}$ and the other two triangles.

From the above similarity results, one can derive the angle and side formulas for $T_{1}$ in terms of the angles and sides of $T_{0}$ as in [Hob97, KS88]. These formulas play a key role in [HZ01] where sequences of pedal triangles are studied in detail. They are also essential in this paper. From the self-similarity property in the construction of pedal triangles, as illustrated in Figure 2, the following angle formulas can be verified immediately. If $T_{0}$ is acute, then

$$
\begin{array}{lll}
A_{1}=\pi-2 A_{0}, & B_{1}=\pi-2 B_{0}, & C_{1}=\pi-2 C_{0} ; \\
a_{1}=a_{0} \cos A_{0}, & b_{1}=b_{0} \cos B_{0}, & c_{1}=c_{0} \cos C_{0} .
\end{array}
$$

Date: May 25, 2005

2000 Mathematics Subject Classification. 40A99, 51A99, 52B60, 28 A80.

Key words and phrases. Sierpiński Triangle, Schur-convex function, pedal triangle. 


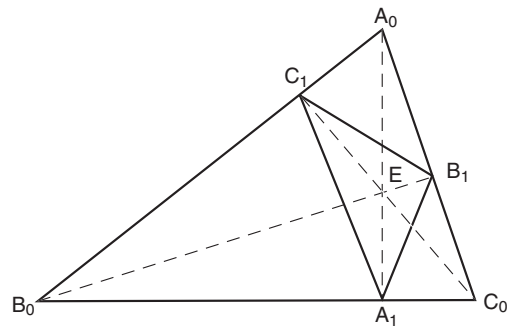

(a)

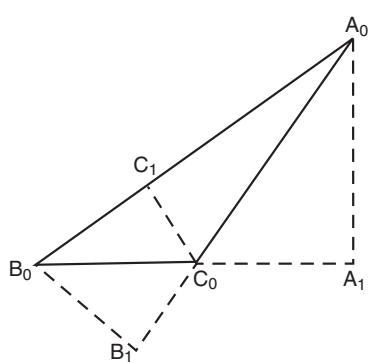

(b)

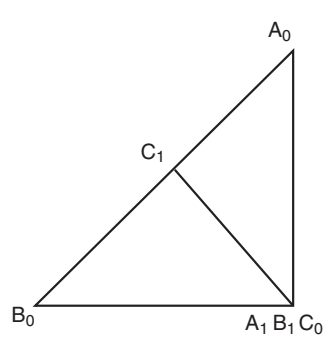

(c)

Figure 1. Pedal Triangle Construction
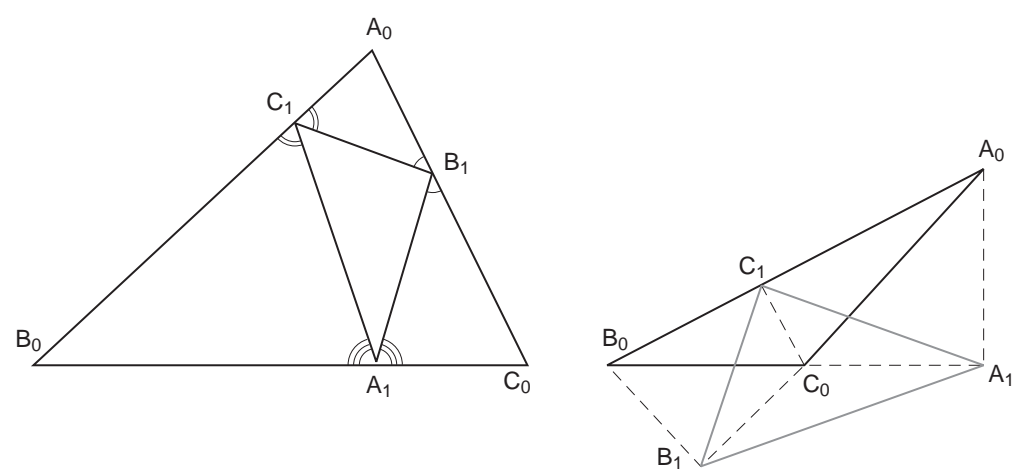

FIGURE 2. Self-Similarity Property of Pedal Triangles

where $a_{0}, b_{0}, c_{0}$, and $a_{1}, b_{1}, c_{1}$ are the sides of $T_{0}$ and $T_{1}$ respectively.

If $T_{0}$ is obtuse with, say, $\pi>A_{0}>\pi / 2$, then

$$
\begin{array}{lll}
A_{1}=2 A_{0}-\pi, & B_{1}=2 B_{0}, & C_{1}=2 C_{0} ; \\
a_{1}=-a_{0} \cos A_{0}, & b_{1}=b_{0} \cos B_{0}, & c_{1}=c_{0} \cos C_{0} .
\end{array}
$$

For an acute triangle $\triangle A_{0} B_{0} C_{0}$, regarding the sides of its pedal triangle $\triangle A_{1} B_{1} C_{1}$ as three light beams and thinking of the sides of $\triangle A_{0} B_{0} C_{0}$ as three mirrors, the similarity between $\triangle A_{0} B_{0} C_{0}$ and each of the three smaller triangles surrounding the pedal triangle $\triangle A_{1} B_{1} C_{1}$ illustrates the optical property that the angle of incidence equals the angle of reflection. Since nature always takes the most economical way, this physical interpretation of pedal triangle also implies an interesting geometric extreme property of pedal triangle called Fagnano's Problem.

Theorem 1.1. [Cox89, p. 20] [CG67, p. 88] For a given acute triangle $\triangle A_{0} B_{0} C_{0}$, the pedal triangle $\triangle A_{1} B_{1} C_{1}$ has the shortest perimeter among all triangles that are inscribed in $\triangle A_{0} B_{0} C_{0}$.

The main purpose of this article is to look at pedal triangles from a different perspective, however. In the next section, we generalize the traditional construction of the Sierpiński triangle to a construction that uses pedal triangles. 


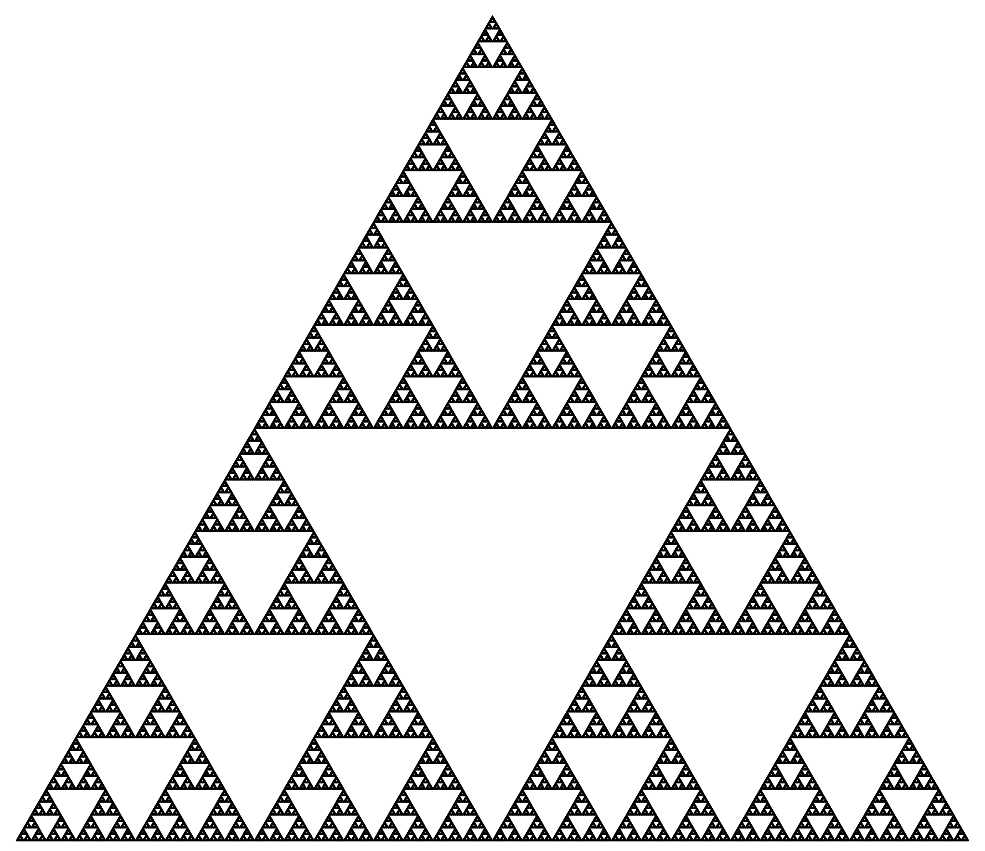

FIGURE 3. Sierpiński Triangle, 10 iterations

We call these objects Sierpiński Pedal Triangles (SPT). In section 3, we compute the area ratios for these SPTs. And in section 4, we show how the fractal dimension of SPT depends on the initial triangle. Section 5 discusses the SPTs from an iterated function system perspective. And in section 6 we discuss the dynamics of the sequence of pedal triangles.

\section{SieRPIŃSKI PEDAL TRIANGLE}

To begin, we recall the construction of the Sierpiński triangle. Let $S_{0}$ be an equilateral triangle (or any other triangle). Joining the middle points on the three sides of $S_{0}$ results a second triangle $T_{0}$ which is similar to $S_{0}$. We call it the middle triangle of $S_{0}$. Let $S_{1}=S_{0} \backslash \operatorname{Int} T_{0}$, i.e., remove the interior of the middle triangle $T_{0}$ from $S_{0}$. Then $S_{1}$ is a union of three smaller triangles each of which is similar to $S_{0}$. From each of these smaller triangles, remove the interior of the middle triangle again, and denote the resulting union of nine equilateral triangles by $S_{2}, S_{2} \subset S_{1}$. Continuing this procedure to define $S_{3} \supset S_{4} \supset S_{5} \cdots$, one obtains the well-known Sierpiński Triangle (ST) by taking the intersection of the nested sequence (see Figure 3).

$$
S T=\bigcap_{n=0}^{\infty} S_{n} .
$$

The Sierpiński triangle can be viewed as one possible generalization of the Cantor set to two-dimensions. Other generalizations are possible. For example, the original triangle $S_{0}$ does not have to be equilateral. It can be any triangle, as long as one joins the middle points from each side to form a middle triangle,

mention these are equivalent via an isometry 


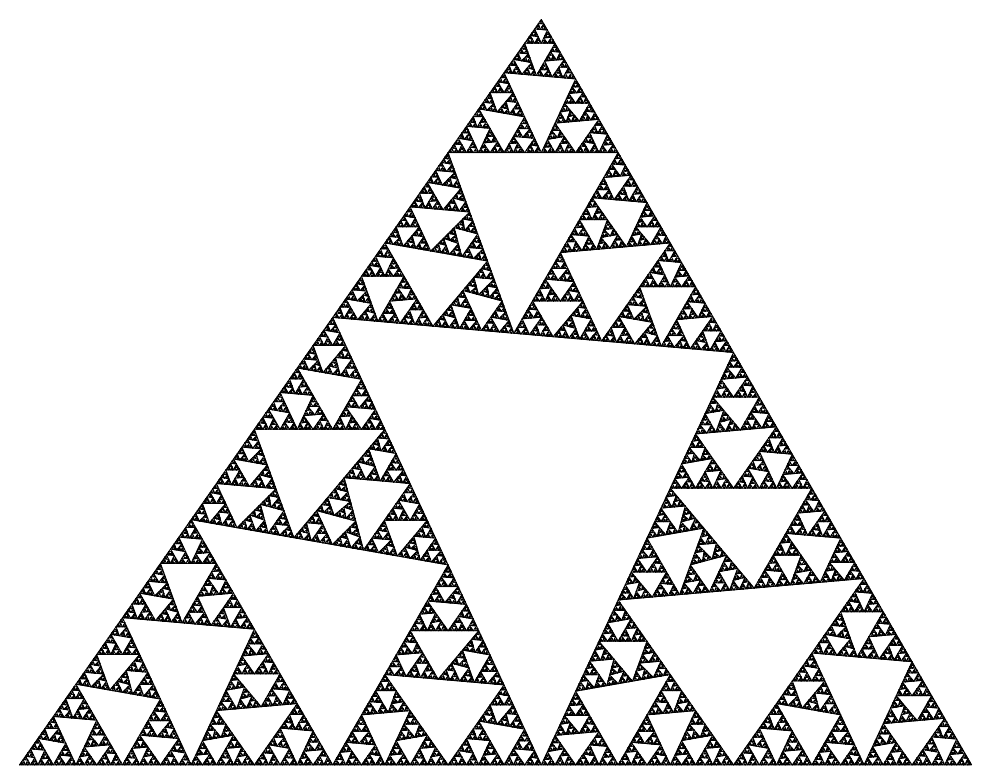

FIGURE 4. SPT constructed from $\left(55^{\circ}-60^{\circ}-65^{\circ}\right)$-triangle, 12 iterations

and removes its interior from the previous triangle. The result will always be three smaller triangles that are similar to their parental triangle. Continuing this process will produce a self-similar fractal. It is natural to ask if these STs are the only self-similar fractal obtained by removing inscribed triangles? That is, for a given triangle $T$, can one remove a triangle whose vertices are on the sides of $T$ and have the resulting three smaller triangles all similar to $T$ ? Well, as we saw in the previous section, the pedal triangle of $T$ is such a triangle. Furthermore, it is the only such triangle other than the ordinary middle triangle. Therefore, it also generates a self-similar fractal, and we call it a Sierpiński pedal triangle (SPT). Figure 4 is an example.

If the initial triangle $T$ is equilateral, then the feet of the three altitudes of $T$ coincide with the middle points of the three sides of $T$, hence the resulting SPT is the same as ST. In this regard, one may view SPT as a generalization of ST.

In the construction of ST, one needs only three contraction maps with the same contraction ratio $1 / 2$. The procedure then iterates to produce ST. In the construction of a SPT, however, one needs not only three contractions maps of (possibly) different contraction ratios, but also reflections. More importantly, the contraction ratios depend upon the shape of the initial parental triangle.

In the remainder of this paper, we examine some fundamental properties of SPT in comparison to ST and see to what extent SPT is a true generalization of ST.

Remark 2.1.

(i) ST and SPT are the only self-similar inscribed triangular fractals. Because the pedal triangle of an obtuse triangle is not inscribed "inside" the parental triangle, from now on, we will be concerned with only acute 

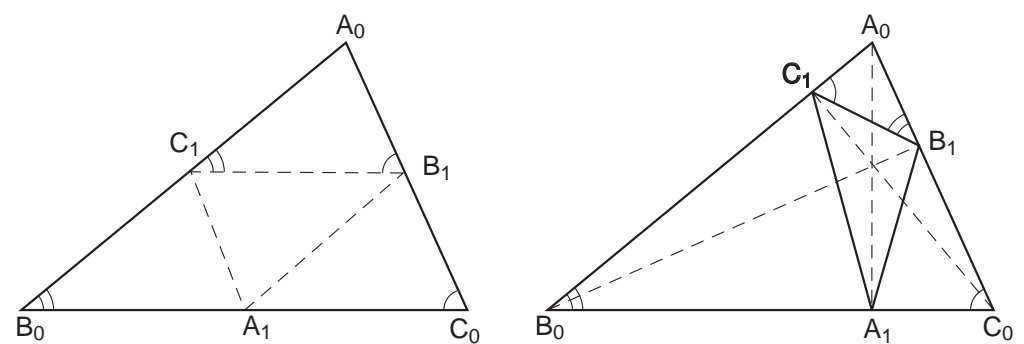

FIGURE 5. Comparison of ST and SPT Constructions

triangles. Let $\triangle A B C$ be any acute triangle and $\triangle A_{1} B_{1} C_{1}$ any inscribed triangle which divides the former into three smaller triangles as shown in Figure 5. If $\triangle A B_{1} C_{1}$ is similar to $\triangle A B C$, then either $\angle B=\angle A C_{1} B_{1}$ and $\angle C=\angle A B_{1} C_{1}$, or $\angle B=\angle A B_{1} C_{1}$ and $\angle C=\angle A C_{1} B_{1}$. The first case can happen only when $\triangle A_{1} B_{1} C_{1}$ is the middle triangle of $\triangle A B C$, and the second case occurs only for the pedal triangle.

(ii) ST and SPT are not affine equivalent. It is clear that when the initial triangle is not equilateral, SPT and ST are not affine equivalent since an affine transformation must preserve the ratio of the line segments on each side of the triangle. Moreover, SPT involves the altitudes, but orthogonality is not an affine invariant. From Figure 5 we can see that for a right triangle, its pedal triangle degenerates to a line segment while the ST is still non-degerate.

\section{The Area Ratio of A Pedal Triangle}

One of the important ways to distinguish two different fractals is to compare their fractal dimensions. Before we consider the fractal dimension of SPT, let us look at the area ratio of a pedal triangle $\triangle A_{1} B_{1} C_{1}$ with its parental triangle $\triangle A B C$, and provide some additional results from the classical geometry of triangles. For the ST case, it is easy to see that the area ratio is always $1 / 4$ because the contraction ratio is $1 / 2$. Then the fractal dimension of ST is easily calculated to be $\ln 3 / \ln 2$. On the other hand, for the SPT case, a direct calculation from formulas $1 \mathrm{a}$ and $1 \mathrm{~b}$ shows that

$$
\frac{\text { Area } \triangle A_{1} B_{1} C_{1}}{\text { Area } \triangle A B C}=\frac{\cos A \cos B \sin (\pi-2 C)}{\sin C}=-2 \cos A \cos B \cos (A+B) \text {, }
$$

where $0<A, B<\pi / 2$ and $\pi / 2<A+B<\pi$.

If we are concerned with only acute triangles, we may introduce the following index domain, $I=\{(x, y) \mid 0<x<\pi / 2,0<y<\pi / 2, \pi / 2<x+y\}$. In Figure 6 , the index domain $I$ is the interior of the center shaded triangle $M_{0}$. The interiors of the three triangles $M_{1}, M_{2}$, and $M_{3}$ surrounding $M_{0}$ represent all possible obtuse triangles. The boundaries between $M_{0}$ and the $M_{i}$ 's, $i=1,2,3$, represent all right triangles. For each $(x, y) \in I$, set $z=\pi-(x+y)$. Then the ordered triple of real numbers $(x, y, z)$ represents an acute triangle in the Euclidean plane with inner angles $x, y$ and $z$.

We will need the following definition. 


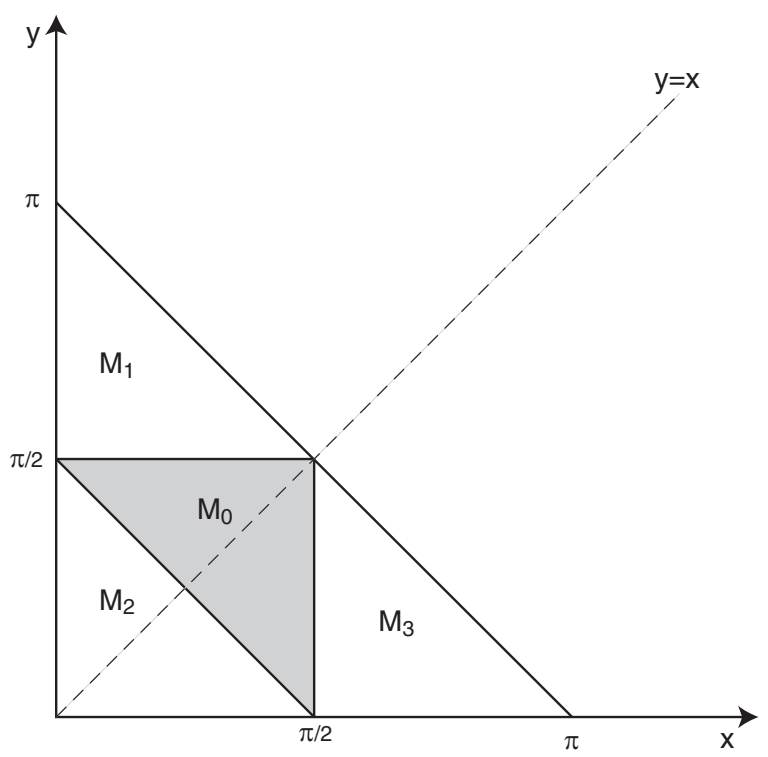

FiguRE 6. The Index Domain

Definition 3.1. A function $f: I \rightarrow \mathbb{R}(n>1)$ is called Schur-convex if for every doubly stochastic matrix $S$,

$$
f(S \mathbf{x}) \leq f(\mathbf{x})
$$

for all $\mathbf{x} \in I$. It is called strictly Schur-convex if the inequality is strict and Schurconcave (respectively, strictly Schur-concave) if the inequality (2) is reversed.

Next we will show that there is a two-parameter family of Sierpiński pedal triangles that can be indexed by points in $I$. Their area ratio is a Schur-concave function on $I$ and their Hausdorff dimension can be described as a function on $I$ as well. To this end, for $(x, y) \in I$, define

$$
f(x, y)=-2 \cos x \cdot \cos y \cdot \cos (x+y) .
$$

It is clear that $f$ is symmetric and differentiable on $I$. Moreover

so

$$
\begin{aligned}
& f_{x}=2 \sin x \cos y \cos (x+y)+2 \cos x \cos y \sin (x+y), \\
& f_{y}=2 \cos x \sin y \cos (x+y)+2 \cos x \cos y \sin (x+y),
\end{aligned}
$$

$$
f_{x}-f_{y}=2 \cos (x+y) \cdot \sin (x-y) .
$$

Therefore, for any $(x, y) \in I$ with $x \neq y$ we always have

$$
\left(f_{x}-f_{y}\right) \cdot(x-y)=2[\cos (x+y) \sin (x-y)] \cdot(x-y)<0
$$

because $\cos (x+y)<0$ and $[\sin (x-y)] \cdot(x-y)>0$ on $I$. But $\left(f_{x}-f_{y}\right)$. $(x-y)<0$ for all $x \neq y$ is equivalent to $f$ being a strict Schur-concave function on $I$. From the special properties of Schur-concave functions (see [MO79, RV73, Zha98]), if $f(x, y)$ has maximum in $I$, it can occur only in the subset of $I$ where $x=y$. That is, $f(x, y)$ attains its maximum only along the 


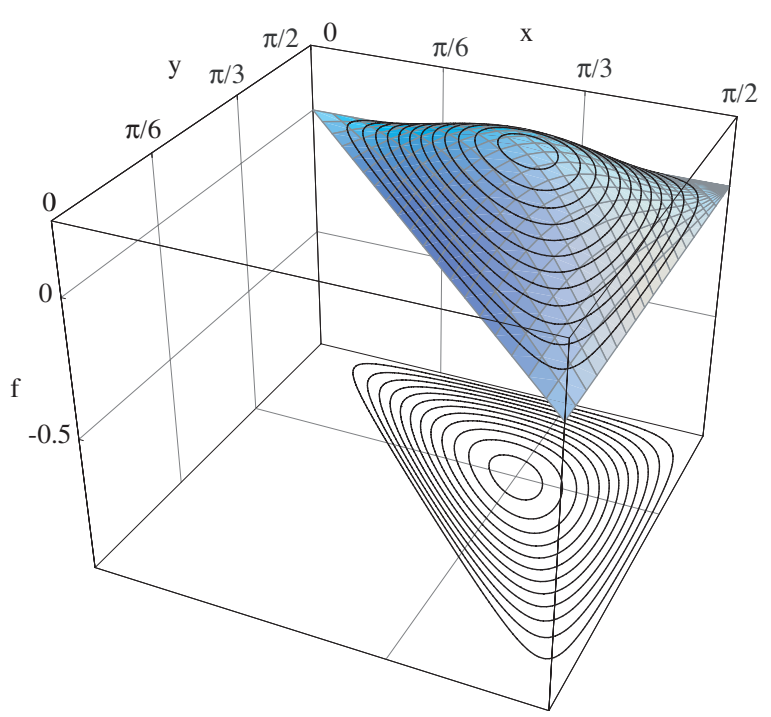

FIGURE 7. Area Ratios for Sierpiński Triangles

intersection of $I$ with the main diagonal of the $x y$-plane. This can also be seen by directly setting $f_{x}=0$ and $f_{y}=0$ and solving the system simultaneously.

Now, consider $f$ along the line $x=y$. It reduces to

$$
f(x)=-2 \cos ^{2} x \cos 2 x=2 \cos ^{2} x-4 \cos ^{4} x .
$$

It is a simple calculation in calculus to find that $f$ has only one critical point $x_{0}=\pi / 3$, and $f$ attains the maximum there.

We define an order " $\prec$ " on $I$ as follows.

Definition 3.2. We say $\left(x_{1}, y_{1}\right) \prec\left(x_{2}, y_{2}\right)$ if and only if there is a $p, 0<p<1$, such that

$$
\left[\begin{array}{l}
x_{2} \\
y_{2}
\end{array}\right]=\left[\begin{array}{cc}
p & 1-p \\
1-p & p
\end{array}\right]\left[\begin{array}{l}
x_{1} \\
y_{1}
\end{array}\right] .
$$

That is, $\left(x_{2}, y_{2}\right)$ can be obtained from $\left(x_{1}, y_{1}\right)$ via multiplication by a $2 \times 2$ doubly stochastic matrix. Equivalently, $\left(x_{2}, y_{2}\right)$ lies in the interior of the line segment between $\left(x_{1}, y_{1}\right)$ and $\left(y_{1}, x_{1}\right)$.

We can summarize the above arguments with the following theorem.

Theorem A. Every similarity class of acute triangles can be represented by a point $(x, y)$ in the index domain I. Furthermore,

(i) $f\left(\frac{\pi}{3}, \frac{\pi}{3}\right)=\frac{1}{4}$ is the maximum;

(ii) $f(x, y) \rightarrow 0$ as $(x, y) \rightarrow \partial M_{0}$;

(iii) $f\left(x_{1}, y_{1}\right)<f\left(x_{2}, y_{2}\right)$ if $\left(x_{1}, y_{1}\right) \prec\left(x_{2}, y_{2}\right)$.

So given any acute triangle, the area ratio of its pedal triangle with the original triangle depends on the shape of the parental triangle. The closer the parental triangle is to being equilateral, the larger the area ratio. This is illustrated in Figure 7, which was generated with Mathematica. 


\section{FRACTAL DIMENSION OF SPT}

In general, the computation of the fractal dimension of a set can be very complex and difficult. But for a self-similar fractal set, its fractal dimension can be calculated by the following useful formula [Bar89, Har89],

$$
\sum_{i=1}^{n} r_{i}^{D}=1
$$

where $n$ is the number of the self-similar pieces reproduced in each step in the construction of a fractal, and the $r_{i}$ 's are the contraction ratios (or magnification factors) for $i=1,2, \cdots, n$ and $D$ is its fractal dimension.

For the case of ST, $n=3, r_{1}=r_{2}=r_{3}=1 / 2$, and the above equation easily implies that $D=\ln 3 / \ln 2$. This is perhaps the simplest and most useful formula to find the Hausdorff dimensions of self-similar fractal sets with constant contraction ratios. However, for the SPT case, $r_{1}, r_{2}$ and $r_{3}$ are different, in general. Solving that algebraic equation for $D$ as a function of $r_{1}, r_{2}$ and $r_{3}$ is not a simple task.

Again, let $\triangle A B C$ be an acute triangle and $\triangle A_{1} B_{1} C_{1}$ its pedal triangle. From formula (1b) we see that the three contraction ratios of the smaller triangles are $r_{1}=\cos A, r_{2}=\cos B$, and $r_{3}=\cos C$. Therefore, the fractal dimension $D$ of SPT associated with $\triangle A B C$ is determined by

$$
\cos ^{D} A+\cos ^{D} B+\cos ^{D} C=1 .
$$

In particular, the fractal dimension of SPT depends on the initial triangle.

\section{Example 4.1.}

(i) If $A=B=C=\pi / 3, \triangle A B C$ is an equilateral triangle, and the SPT is the same as the ST. So $D=\ln 3 / \ln 2 \approx 1.58496$.

(ii) $A=\pi / 3, B=\pi / 4$, and $C=5 \pi / 12$, we have

$$
\left(\frac{1}{2}\right)^{D}+\left(\frac{\sqrt{2}}{2}\right)^{D}+\left(\cos \frac{5 \pi}{12}\right)^{D}=1 .
$$

Solving this numerically yields $D \approx 1.63343$.

(iii) $A=\pi / 2, B=\pi / 2-C$, a right triangle, then equation 3 becomes

$$
0^{D}+\cos ^{D} B+\sin ^{D} B=1
$$

which implies $D=2$.

Let $D(x, y)$ denote the fractal dimension of the SPT generated by an acute triangle represented by a point $(x, y)$ in the index domain $I$. We have

Theorem B. $D(x, y)$ attains an absolute minimum value of $\frac{\ln 3}{\ln 2}$ on the index domain $I$ at the point $(\pi / 3, \pi / 3)$.

Proof. First we will show that $D(x, y)$ has a relative minumum at $(\pi / 3, \pi / 3)$. Rewriting equation 3 as

$$
\cos ^{D} x+\cos ^{D} y+\cos ^{D}(z)=1
$$

where $z=\pi-x-y$ and differentiating implicitly, we obtain

$$
\begin{aligned}
& {\left[\cos ^{D} x \cdot \ln (\cos x)+\cos ^{D} y \cdot \ln (\cos y)+\cos ^{D} z \cdot \ln (\cos z)\right] \cdot D_{x}=} \\
& \cos ^{D} x \cdot D \cdot \tan x-\cos ^{D} z \cdot D \cdot \tan z, \text { and }
\end{aligned}
$$




$$
\begin{aligned}
& {\left[\cos ^{D} x \cdot \ln (\cos x)+\cos ^{D} y \cdot \ln (\cos y)+\cos ^{D} z \cdot \ln (\cos z)\right] \cdot D_{y}=} \\
& \cos ^{D} y \cdot D \cdot \tan y-\cos ^{D} z \cdot D \cdot \tan z .
\end{aligned}
$$

Since the coefficients of $D_{x}$ and $D_{y}$ in the above equations can never be zero for $(x, y) \in I$, we have $D_{x}=D_{y}=0$ if and only if

$$
\begin{aligned}
& \cos ^{D} x \cdot D \cdot \tan x-\cos ^{D} z \cdot D \cdot \tan z=0, \quad \text { and } \\
& \cos ^{D} y \cdot D \cdot \tan y-\cos ^{D} z \cdot D \cdot \tan z=0 .
\end{aligned}
$$

An easy check shows that $D_{x}(\pi / 3, \pi / 3)=D_{y}(\pi / 3, \pi / 3)=0$, i.e., $(\pi / 3, \pi / 3)$ is a critical point of $D(x, y)$ inside the index domain $I$. We can find the second order partial derivatives of $D$ via implicit differentiation on equations 4 and 5. Through a lengthy but direct calculation, we find that at the point $(x, y)=$ $(\pi / 3, \pi / 3)$

$$
\begin{aligned}
&\left|\begin{array}{cc}
D_{x x} & D_{x y} \\
D_{y x} & D_{y y}
\end{array}\right|=\left|\begin{array}{cc}
2\left(\log _{2} 3-4 / 3\right) & \log _{2} 3-4 / 3 \\
\log _{2} 3-4 / 3 & 2\left(\log _{2} 3-4 / 3\right)
\end{array}\right|\left(\frac{\ln 3}{(\ln 2)^{2}}\right)^{2}= \\
& 3\left(\log _{2} 3-\frac{4}{3}\right)^{2}\left[\frac{\ln 3}{(\ln 2)^{2}}\right]^{2}>0
\end{aligned}
$$

This shows that $(\pi / 3, \pi / 3)$ is a relative minumum of $D$ on $I$.

To see that $(\pi / 3, \pi / 3)$ is the absolute minimum on $I$, we will show it is the only critical point on $I$. To do this, assume that $(a, b)$ is a critical point on $I$ with $D_{x}(a, b)=0=D_{y}(a, b)$. Then equations 4 and $5 \operatorname{imply} \cos ^{D} a \cdot \tan a=$ $\cos ^{D} b \cdot \tan b$. Then

$$
\left(\frac{\cos a}{\cos b}\right)^{D}=\frac{\tan b}{\tan a} .
$$

Solving for $D$, and assuming $x \neq y$, gives

$$
D(a, b)=\frac{\ln \left(\frac{\tan b}{\tan a}\right)}{\ln \left(\frac{\cos a}{\cos b}\right)} .
$$

Next, we note that $D(x, y)$ possesses a six-fold symmetry over $I$. This is seen by the fact that the fractal dimension of a triangle with angles $(x, y, \pi-$ $x-y)$ can be computed on $I$ using any two of the three angles in either order: $D(x, y)=D(y, x)=D(x, \pi-x-y)=D(\pi-x-y, x)=D(y, \pi-x-y)=$ $D(\pi-x-y, y)$.

STILL NEEDS WORK

This completes the proof of Theorem B.

Let $(x, y)$ be a point in the index domain $I$ and let $D(x, y)$ be the fractal dimension of SPT generated by the triangle with inner angles $x, y$, and $\pi-$ $(x+y)$. We have the following two conjectures.

Theorem C. With notation as above,

$$
\frac{\ln 3}{\ln 2} \leq D(x, y)<2
$$

Conjecture D. With notation as above, $D\left(x_{1}, y_{1}\right) \leq D\left(x_{2}, y_{2}\right)$ if $\left(x_{2}, y_{2}\right) \prec$ $\left(x_{1}, y_{1}\right)$ for any $\left(x_{1}, y_{1}\right),\left(x_{2}, y_{2}\right) \in I$.

Remark 4.2. 


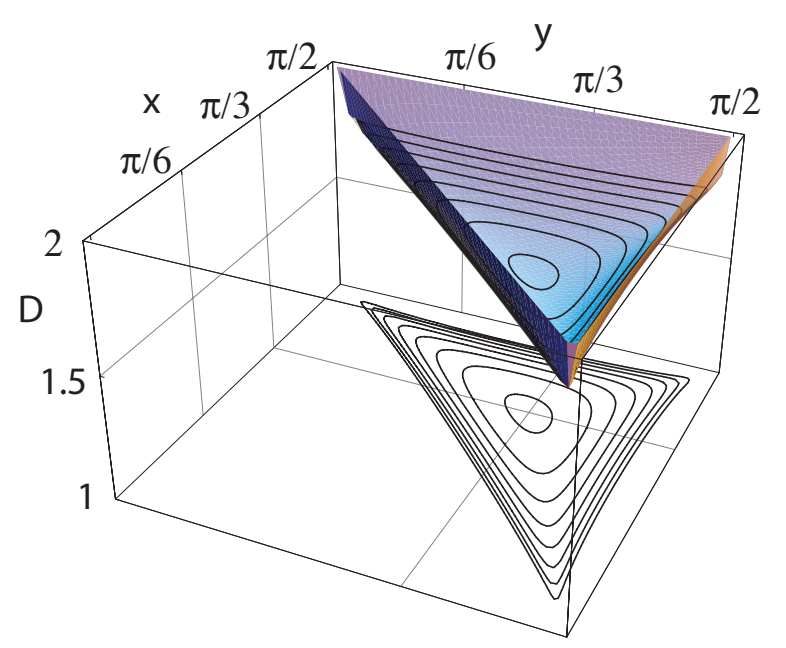

FIGURE 8. The Dimension, $D(x, y)$, of SPT on $I$

(i) Theorem C follows immediately from Theorem B since $D(\pi / 3, \pi / 3)$ is a global minimum.

(ii) Conjecture $\mathrm{D}$ claims that $D(x, y)$ is a Schur-convex function on $I$. The function $D(x, y)$ is illustrated in Figure 8.

(iii) Conjecture $\mathrm{D}$ is supported by the data shown in Figure 8, which was generated with Mathematica. That is, the more a triangle $(x, y)$ deviates from the equilateral triangle $\left(\frac{\pi}{3}, \frac{\pi}{3}\right)$, the bigger its fractal dimension $D(x, y)$. Some specific calculations are contained in Table 1.

\begin{tabular}{||c|c||}
\hline Triangle & $D(x, y)$ \\
\hline$\left(25^{\circ}, 75^{\circ}, 80^{\circ}\right)$ & 1.875 \\
\hline$\left(35^{\circ}, 65^{\circ}, 80^{\circ}\right)$ & 1.6875 \\
\hline$\left(45^{\circ}, 60^{\circ}, 75^{\circ}\right)$ & 1.6337 \\
\hline$\left(50^{\circ}, 60^{\circ}, 70^{\circ}\right)$ & 1.6208 \\
\hline$\left(55^{\circ}, 60^{\circ}, 65^{\circ}\right)$ & 1.611237 \\
\hline$\left(60^{\circ}, 60^{\circ}, 60^{\circ}\right)$ & 1.584796 \\
\hline
\end{tabular}

TABLE 1: Triangles And The Fractal Dimensions Of Their SPT

Figures 9, 10, 11, 12, 4, and 3 illustrate the Sierpiński pedal triangles corresponding, respectively, to the entries in the table. Entry six in the table is the Sierpiński triangle already shown in Figure 3. These images were produced with Mathematica using a modification of code for producing Sierpiński triangles found in [Wag00].

(iv) If the initial triangle $\triangle A B C$ is obtuse, then it generates a self-similar fractal SPT with overlaps. One may still talk about its fractal dimension, but more detailed discussion about Hausdorff measure theory is required. Interested readers may refer to [Mat95].

In summary, we may view SPT as a natural generalization of ST, and represent SPT as a two-parameter family of fractal sets constructed from triangles.

Moreover, for the roles played by ST in different circumstances such as in 


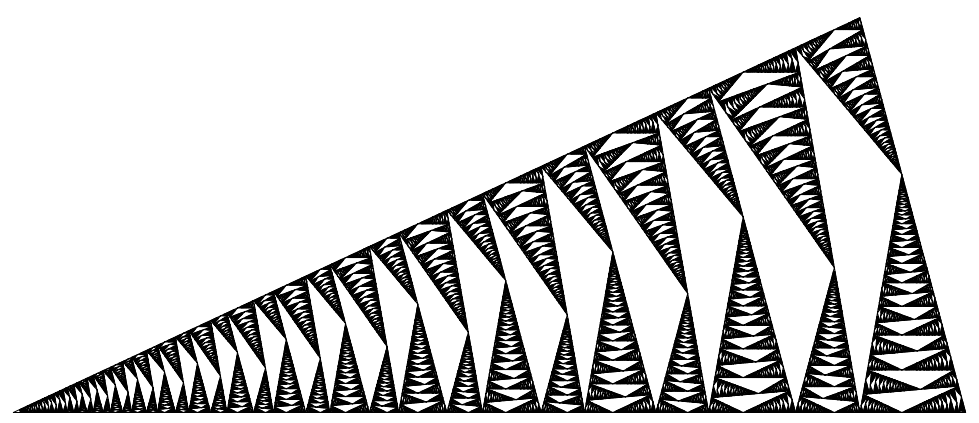

FIGURE 9. $25^{\circ}-75^{\circ}-80^{\circ}$ Triangle, 50 Iterations

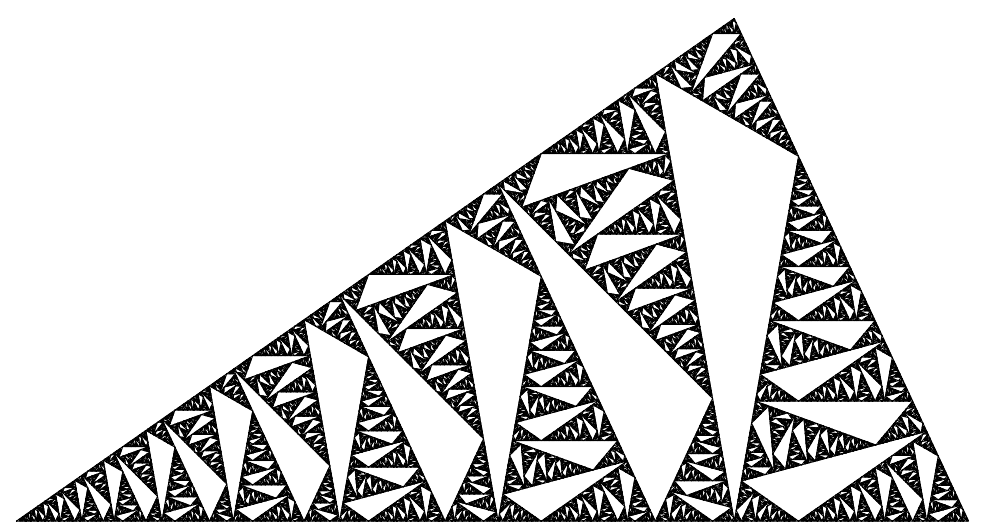

FiguRE 10. $35^{\circ}-65^{\circ}-80^{\circ}$ Triangle, 30 Iterations

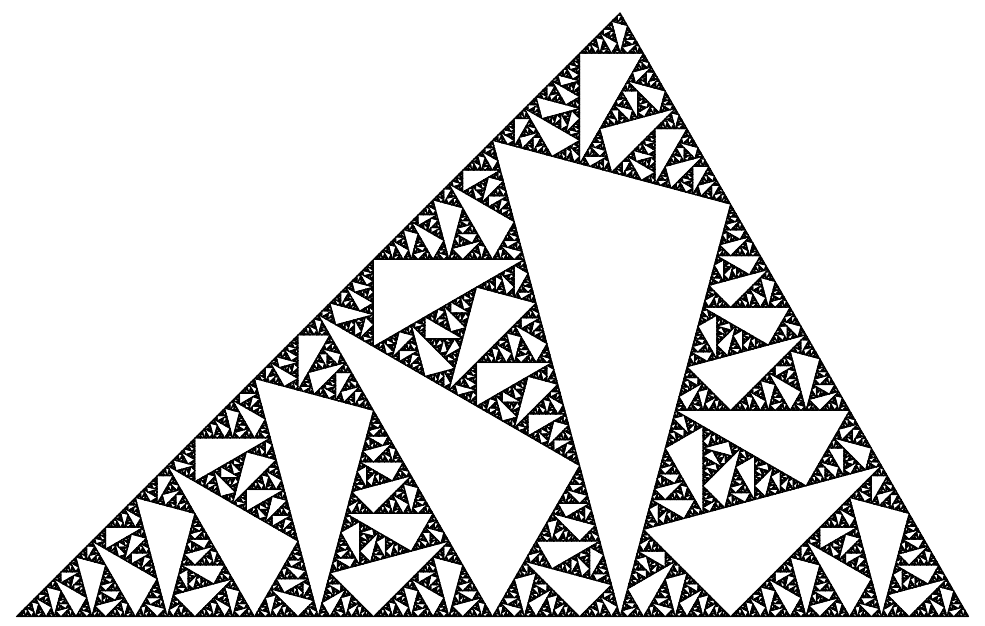

FIGURE $11.45^{\circ}-60^{\circ}-75^{\circ}$ Triangle, 20 Iterations 


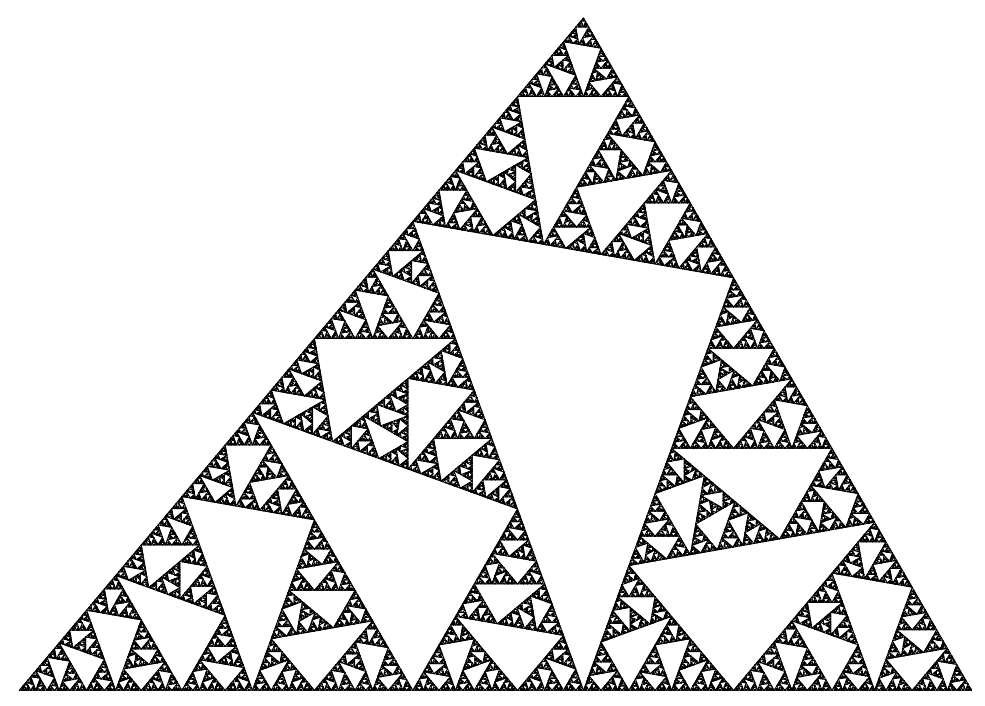

FIGURE $12.50^{\circ}-60^{\circ}-70^{\circ}$ Triangle, 15 Iterations

chaos games and in IFS [Bar89, Man82, Ste95], we hope that SPT can be introduced naturally into different areas of studies as a generalization of ST.

\section{ITERATED FUNCTION SYSTEMS}

Insert explanation for Figure 13.

\section{Sequences of Pedal Triangles}

this repeats the introduction

Another interesting property of pedal triangles is that they can form natural sequences in which the shapes change chaotically. Let $T_{0}$ be a triangle with inner angles $A_{0}, B_{0}$, and $C_{0}$. Construct a second triangle $T_{1}$ with inner angles $A_{1}, B_{1}$, and $C_{1}$ whose vertices are the feet of the three altitudes of $T_{0}$. Construct a third triangle $T_{2}$ with inner angles $A_{2}, B_{2}$, and $C_{2}$ whose vertices are the feet of the three altitudes of $T_{1}$. Construct a triangle $T_{3}$ from $T_{2}$ in the same way, and so on. One obtains a sequence of triangles $\left\{T_{n}\right\}_{n=0}^{\infty}$ where $T_{n+1}$ is the pedal triangle of $T_{n}$. It is called the sequence of pedal triangles generated by $T_{0}$. See Figure 14.

While the size of these pedal triangles gets smaller rapidly, an interesting question is: "what can we say about the change of their shapes?" This problem and questions involving limiting shape of different sequences of plane triangles have been studied since at least a century ago [Hob97]. In late 1980's, Kingston and Synge revisited the sequence of pedal triangles problem [KS88]. They discovered many interesting properties of such sequences and also fixed some errors that occured in the earlier literature. Soon after their work, a number of articles made nice connections between the sequence of pedal triangles and symbolic dynamic systems and ergodic theory [Ale93, Hob97, Lax90, MO79, Ung90]. It seems that many fundamental concepts and phenomenon in the study of dynamic systems can be found in the sequence of pedal triangles. As Alexander 

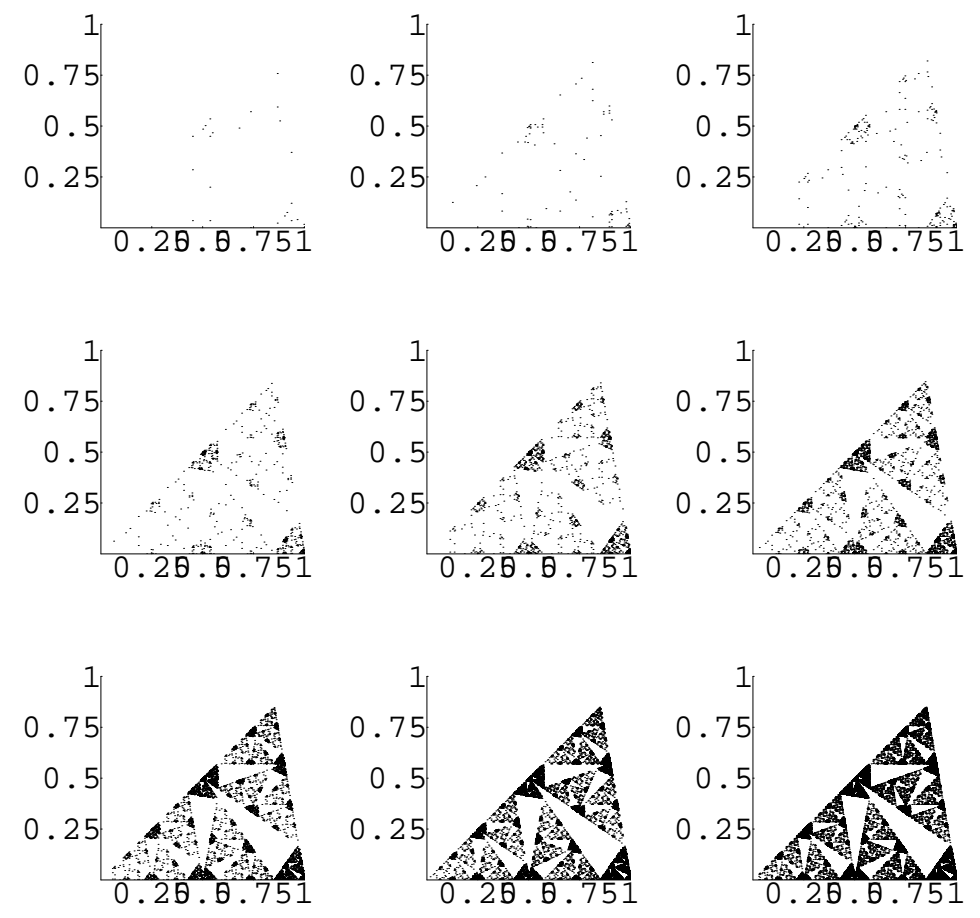

FIGURE 13 . SPT $45^{\circ}-55^{\circ}-80^{\circ}$ Triangle using IFS

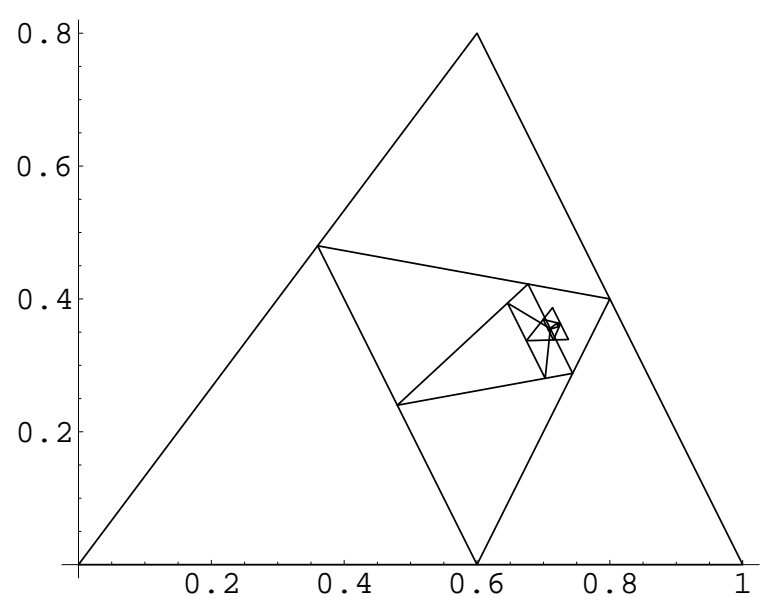

FIGURE 14. A Sequence of Pedal Triangles

pointed out that one could use the special properties of these pedal triangles as an elementary and expository introduction to the power and the beauty of symbolic dynamic systems [Ale93]. On the other hand, the well established theory 
of symbolic dynamic systems, ergodic theory, fractal geometry, and computer graphics will no doubt provide powerful new tools for many classical geometry problems that have proven difficult to tackle using the classical synthetic methods. In [DHZ03, HZ01], we investigated some interesting dynamic systems problems in classical geometry. Different sequences of triangles always provided important and inspiring examples. In particular, the chaotic behavior of the sequence of pedal triangles motivate people to further explore the possible intrinsic connections between classical geometry problems and some advanced mathematical theories. It is interesting to note that when a dynamic system proved to be chaotic, fractal appears in one way or another although the two subjects are completely independent from each other. Most textbooks of dynamic systems treat fractals as the strange attractors, but fractals occur everywhere. To conclude this article, we like to ask: Is the fractal dimension of a Sierpiński pedal triangle generated by a given triangle $T_{0}$ related to chaotic property of the sequence of pedal triangles generated by $T_{0}$ ?

\section{REFERENCES}

[Ale93] J. C. Alexander, The symbolic dynamics of the sequence of pedal triangles, Math. Mag. 66 (1993), no. 3, 147-158. 12, 13

[Bar89] Michael F. Barnsley, Lecture notes on iterated function systems, Chaos and fractals (Providence, RI, 1988), Proc. Sympos. Appl. Math., vol. 39, Amer. Math. Soc., Providence, RI, 1989, pp. 127-144. 8, 12

[CG67] H. S. M. Coxeter and S. L. Greitzer, Geometry revisited, Math. Assoc. Amer., 1967. 2

[Cox89] H. S. M. Coxeter, Introduction to geometry, John Wiley \& Sons Inc., New York, 1989, Reprint of the 1969 edition. 2

[DHZ03] Jiu Ding, L. Richard Hitt, and Xin-Min Zhang, Markov chains and dynamic geometry of polygons, Linear Algebra Appl. 367 (2003), 255-270. 14

[Har89] Jenny Harrison, An introduction to fractals, Chaos and fractals (Providence, RI, 1988), Proc. Sympos. Appl. Math., vol. 39, Amer. Math. Soc., Providence, RI, 1989, pp. 107-126. 8

[Hob97] E. W. Hobson, A treatise on plane trigonometry, Cambridge University Press, 1897. 1, 12

[HZ01] L. Richard Hitt and Xin-Min Zhang, Dynamic geometry of polygons, Elem. Math. 56 (2001), no. 1, 21-37. 1, 14

[KS88] John G. Kingston and John L. Synge, The sequence of pedal triangles, Amer. Math. Monthly 95 (1988), no. 7, 609-620. 1, 12

[Lax90] Peter D. Lax, The ergodic character of sequences of pedal triangles, Amer. Math. Monthly 97 (1990), no. 5, 377-381. 12

[Man82] Benoit B. Mandelbrot, The fractal geometry of nature, W. H. Freeman and Co., San Francisco, Calif., 1982, Schriftenreihe für den Referenten. [Series for the Referee]. 12

[Mat95] Pertti Mattila, Geometry of sets and measures in Euclidean spaces, Cambridge University Press, Cambridge, 1995, Fractals and rectifiability. 10

[MO79] Albert W. Marshall and Ingram Olkin, Inequalities: theory of majorization and its applications, Academic Press Inc. [Harcourt Brace Jovanovich Publishers], New York, 1979. 6,12

[RV73] A. Wayne Roberts and Dale E. Varberg, Convex functions, Academic Press [A subsidiary of Harcourt Brace Jovanovich, Publishers], New York-London, 1973, Pure and Applied Mathematics, Vol. 57. 6

[Ste95] Ian Stewart, Four encounters with Sierpiński's gasket, Math. Intelligencer 17 (1995), no. 1, 52-64. 12 
[Ung90] Peter Ungar, Mixing property of the pedal mapping, Amer. Math. Monthly 97 (1990), no. $10,898-900.12$

[Wag00] Stan Wagon, Mathematica in action, second ed., Springer-Verlag, 2000. 10

[Zha98] Xin-Min Zhang, Schur-convex functions and isoperimetric inequalities, Proc. Amer. Math. Soc. 126 (1998), no. 2, 461-470. 6

E-mail address: jding@yizhi .usm.edu

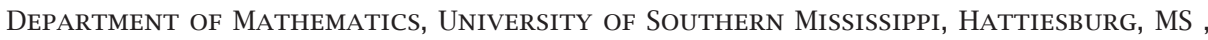
USA

E-mail address: richard@rhitt.com

Department of Mathematics and Statistics, University of South Alabama, Mobile, AL 36688, USA

E-mail address: zhang@jaguar1.usoutha1.edu

Department of Mathematics and Statistics, University of South Alabama, Mobile, AL 36688, USA 